\title{
Signal Power Variation with Applications to Cognitive Radio
}

\author{
Peter J. Smith*, Himal A. Suraweera ${ }^{\dagger}$ and Mansoor Shafi ${ }^{\ddagger}$ \\ * Department of Electrical and Computer Engineering, University of Canterbury \\ Private Bag 4800, Christchurch, New Zealand \\ ${ }^{\dagger}$ Centre for Telecommunications and Microelectronics, School of Electrical Engineering \\ Victoria University, PO Box 14428, Melbourne, Victoria 8001, Australia \\ $\ddagger$ Telecom New Zealand, PO Box 293, Wellington, New Zealand \\ e-mail: p.smith@elec.canterbury.ac.nz,himal.suraweera@vu.edu.au,mansoor.shafi@telecom.co.nz
}

\begin{abstract}
The performance of cognitive radio systems will depend critically on the ability of the secondary user to relinquish borrowed spectrum when it's interference level at the primary user exceeds a certain threshold. Hence, in this paper we investigate the temporal behaviour of this interference power. In particular, we derive the level crossing rate (LCR) of the power. This gives the rate at which a cognitive radio's interference level increases above the acceptable threshold. The LCR is derived for the case of lognormal shadow fading and two different mobility models. The results cater for temporal variations in both shadowing and distance. The basic nature of the LCR appears to be insensitive to the mobility model and suggests that a simple Gaussian LCR can be used as a good approximation in a broad range of cases.
\end{abstract}

Index Terms-Cognitive radio, shadow fading, level crossing rate, spatial shadowing, lognormal processes.

\section{INTRODUCTION}

Radio spectrum is one of the most scarce and valuable resources for wireless communications. Conservative spectrum policies employed by regulatory authorities have resulted in spectrum congestion and underutilization of the overall available spectrum for communications. Measurements performed by agencies such as the Federal Communications Commission [1] in the United States and Ofcom [2] in the United Kingdom have revealed that at any given time, large portions of spectrum are sparsely occupied. Findings of such campaigns on spectrum usage have challenged the traditional spectrum management approaches.

The concept of cognitive radio (CR) first introduced by Mitola [3] refers to a smart radio which can sense the external electromagnetic environment and adapt its transmission parameters according to the current state of the environment [4]. CRs employing opportunistic spectrum access can access parts of the spectrum for their information transmission, provided that they cause minimal interference to the primary users in that band [5], [6]. Therefore, spectrum sharing among the primary licensee and the secondary CR must be carried out in a controlled fashion [7].

Consider a $\mathrm{CR}$ that senses the spectrum before transmission. In this phase of the CR operation, the spectrum sensing is likely to be accurate as the terminal is able to allocate a reasonable amount of time to signal detection. Once the CR has an established link, repeated checking of the frequency band in use will occur. However, this monitoring will only be for short periods of time so as not to interrupt transmission. As a result, the CR link is likely to be established satisfactorily but changes in the spectrum environment during transmission may cause problems. Hence it is important to assess the rate of change of the spectrum environment. Here, there are two types of changes. The first kind is a change of state where the primary user (PU) either takes or drops channels in the band used by the CR. This is governed by the activity factor of the PU. The second kind relates to changes in the signal strength. For example, the PU may have been shadowed from the CR at call initiation but mobility may result in an increasing PU signal strength. The CR should detect this and release the borrowed spectrum. Conversely, a CR may be operating within acceptable interference temperature limits but the mobility may cause its interference levels to rise at a particular PU receiver. It is the second type of change we analyze in this work. Hence, we consider the joint effects of shadowing and distance on signal strength variation.

A crude approximation to CR detection of a PU is to set a threshold power [8], [9], below which detection fails and above which detection is certain. Similarly, an interference temperature approach will allow interference up to a certain threshold, but not above. Hence. the level crossing rate (LCR) of signal strength across a given threshold is particularly useful in CR analysis. LCR analysis for Rayleigh fading [10], [11] is very well known and more recently, exact [12] and approximate [13] results have appeared for the LCRs of multiple-input multiple-output (MIMO) eigenvalues. For shadow fading, an excellent LCR analysis is given in [14]. This work evaluates the LCR due to shadowing but does not consider the effect of distance variation on the LCR of signal power. The novelty of this work lies in the joint investigation of temporal changes in both distance and shadowing. Hence, we consider variations in signal strength which include both path loss and shadow fading effects. To the best our knowledge, this is the first investigation of signal power LCR which includes stochastic models for distance and therefore considers path loss variation.

The rest of the paper is organized as follows: In Section II we describe the system model. In Section III we derive 


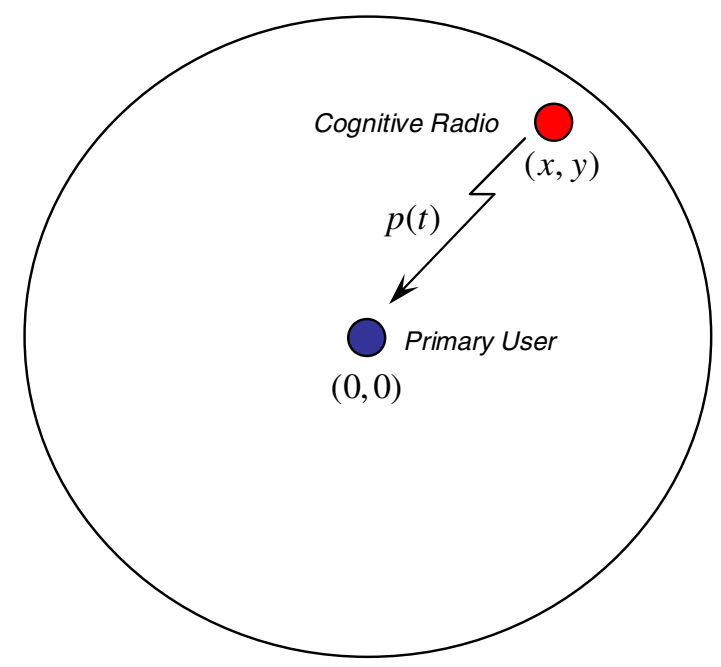

Fig. 1. A fixed PU transmitter with a mobile CR in the vicinity.

the LCR results and in Section IV, analytical and simulation results are presented and discussed. Finally, in Section V some conclusions are given.

\section{System AND MODEL}

Consider a fixed PU transmitter with a mobile CR in the vicinity, as shown in Fig. 1. We locate the PU at the origin and denote the coordinates of the CR at time $t$ as $(x(t), y(t))$. The distance of the CR from the PU is $d(t)=\sqrt{x^{2}(t)+y^{2}(t)}$ and the classical signal strength model is

$$
p(t)=A e^{X(t)}[d(t)]^{-\gamma}
$$

In (1), $p(t)$ is the signal strength of the CR signal received at the PU, $X(t) \sim \mathcal{N}\left(0, \sigma_{s}^{2}\right)$ giving lognormal shadowing and $\gamma$ is the path loss exponent (normally in the range of 2 to 4). The constant $A$ is determined by the transmit power. Note that this problem is defined in terms of the CR signal at the PU, but we could equally well consider the PU signal at the $\mathrm{CR}$. In order for a LCR of $p(t)$ to be valid we must consider stationary models for $X(t)$ and $d(t)$. This is reasonable for short periods of time although in the longer term, directionality of the CR paths will tend to give a non-stationary trend to the distance component. In this paper, we consider simple Gaussian processes for the CR motion. Such models are not particularly realistic but represent a suitable starting point for analysis. Furthermore, results suggest that the nature of the LCR is insensitive to the precise mobility model used.

\section{A. Motion Model 1 (MM1)}

Here the distance, $d(t)$, is assumed to follow a stationary Gaussian process with mean distance $d_{0}$, standard deviation $\sigma_{m}$ and autocorrelation function (ACF) $\rho_{m}(\tau)$. The subscript, $m$, denotes mobility. For small time lags, $\tau \approx 0$, we further assume that $\rho_{m}(\tau)=1-a \tau^{2}+o\left(\tau^{2}\right)$ as in [13] and [15]. By standard usage, $f(\tau)=o\left(\tau^{2}\right)$ is used to indicate that $\lim _{\tau \rightarrow 0}\left|\frac{f(\tau)}{\tau^{2}}\right|=0$. Discrete time simulations of such a model are achieved via

$$
d(t)=d_{0}+\rho_{m}(\tau)\left[d(t-\tau)-d_{0}\right]+\sqrt{1-\rho_{m}^{2}(\tau)} \epsilon(t)
$$

where $\epsilon(t)$ is an independent and identically distributed (i.i.d) $\mathcal{N}\left(0, \sigma_{m}^{2}\right)$ process.

\section{B. Motion Model 2 (MM2)}

Here we assume a more realistic two-dimensional motion where both the $x$ and $y$-coordinates of the $\mathrm{CR}$ vary as stationary Gaussian processes. We adopt some of the notation from MM1 so that the mean position is $\left(x_{0}, y_{0}\right)$, both $x(t)$ and $y(t)$ have standard deviation $\sigma_{m}$ and the common ACF is $\rho_{m}(\tau)$. Discrete time simulations of the CR position follow from

$$
\begin{aligned}
& x(t)=x_{0}+\rho_{m}(\tau)\left(x(t-\tau)-x_{0}\right)+\sqrt{1-\rho_{m}^{2}(\tau)} \epsilon_{x}(t) \\
& y(t)=y_{0}+\rho_{m}(\tau)\left(y(t-\tau)-x_{0}\right)+\sqrt{1-\rho_{m}^{2}(\tau)} \epsilon_{y}(t)
\end{aligned}
$$

where $\epsilon_{x}(t)$ and $\epsilon_{y}(t)$ are i.i.d $\mathcal{N}\left(0, \sigma_{m}^{2}\right)$ processes. With this $2 \mathrm{D}$ model we have the distance process, $d(t)=$ $\sqrt{x^{2}(t)+y^{2}(t)}$.

\section{Shadowing Model}

We assume stationary correlated lognormal shadowing so that $X(t) \sim \mathcal{N}\left(0, \sigma_{s}^{2}\right)$. The ACF is $\rho_{X}(\delta)$ where $\delta$ represents distance. Hence the correlation between $X\left(t_{1}\right)$ and $X\left(t_{2}\right)$ where the spatial separation is $\delta$ is given by $\rho_{X}(\delta)$. Again, we assume that $\rho_{X}(\delta) \approx 1-b \delta^{2}+o\left(\delta^{2}\right)$ when $\delta \approx 0$. Note that this assumption is not satisfied by the well-known Gudmundson model [16]. However, as discussed in [14], there are physical problems with the Gudmundson model, which leads to an infinite LCR. To avoid such difficulites Patzöld and Yang [14] propose alternative models which satisfy the assumption. This is the approach taken here. In the technical literature, lognormal shadowing is usually characterized in terms of its $\mathrm{dB}$ spread which is related to $\sigma_{s}$ by $\sigma_{s}=0.1 \ln (10) \sigma_{\mathrm{dB}}$. As indicated by empirical measurements, values of the $\mathrm{dB}$ spread for wireless systems typically range from 6 to $12 \mathrm{~dB}$ [17].

For MM2 we compute spatial correlation via the Euclidean distance between the $(x, y)$ coordinates at two time points. For MM1, which is essentially a one-dimensional model, we use $d\left(t_{2}\right)-d\left(t_{1}\right)$ to give a measure of spatial separation.

\section{ANALYSIS}

Changes in $p(t)$, particularly relative to an interference temperature threshold, are of interest. Hence we consider the LCR of $p(t)$ across $\tilde{T}$, denoted by $\operatorname{LCR}_{p}(\tilde{T})$. Recalling that $p(t)$ is $A e^{X(t)} d(t)^{-\gamma}$ and taking logarithms, we see that

$$
\operatorname{LCR}_{p}(\tilde{T})=\operatorname{LCR}_{g}(T)
$$

where $g(t)$ is the process

$$
g(t)=X(t)-\gamma \log (d(t))
$$

and $T=\log (\tilde{T})-\log (A)$. The classic Rice formula for the LCR [11] is given by

$$
\operatorname{LCR}_{g}(T)=\int_{0}^{\infty} \dot{g} f_{g, \dot{g}}(T, \dot{g}) d \dot{g}
$$


where $\dot{g}(t)$ is the derivative of $g(t)$ and $f_{g, \dot{g}}(\cdot, \cdot)$ is the joint density of $g(t)$ and $\dot{g}(t)$. This joint density can be broken down as below

$$
f_{g, \dot{g}}(T, \dot{g})=\int_{0}^{\infty} f_{g}(T \mid d=z) f_{\dot{g}}(\dot{g} \mid d=z) f_{d}(z) d z
$$

where $f_{g}(\cdot), f_{\dot{g}}(\cdot)$ and $f_{d}(\cdot)$ are the probability density functions (PDFs) of $g(t), \dot{g}(t)$ and $d(t)$ respectively. This result follows since for fixed $d(t)=z, g(t)=X(t)-\gamma \log (d(t))$ and $\dot{g}(t)=\dot{X}(t)-\gamma \dot{d}(t) / d(t)$ are independent of each other. The independence follows from the well-known property that $X(t)$ is independent of $\dot{X}(t)$ [11] and $X(t)$ is also independent of $\dot{d}(t)$ by construction.

\section{A. $M M 1$}

For MM1, $d(t)$ is Gaussian, $d(t) \sim \mathcal{N}\left(d_{0}, \sigma_{m}^{2}\right)$ so $f_{d}(z)$ is a Gaussian PDF. Similarly, for fixed $d(t)=z, g(t)$ is also Gaussian, $g(t) \sim \mathcal{N}\left(-\gamma \log z, \sigma_{s}^{2}\right)$. Hence, it remains to compute the density of $\dot{g}$. Now $\dot{g}(t)$ relies on $\dot{X}(t)$ which is given by

$$
\dot{X}(t)=\frac{\partial d(t)}{\partial t} \frac{\partial X(t)}{\partial d(t)}=\dot{d}(t) \frac{\partial X(t)}{\partial d(t)}
$$

By construction, both $d(t)$ and $X(t)$ are Gaussian processes and their derivatives are therefore independent Gaussian processes [11]. We write $\dot{d}(t)=\epsilon_{d}(t)$ and $\frac{\partial X(t)}{\partial d(t)}=\epsilon_{X}(t)$ where $\epsilon_{d}(t), \epsilon_{X}(t)$ are white Gaussian processes with mean zero and variances $2 a \sigma_{m}^{2}$ and $2 b \sigma_{s}^{2}$ respectively. With this notation, $\dot{g}(t)=\epsilon_{d}(t)\left[\epsilon_{X}(t)-\gamma / d(t)\right]$, and the conditional density of $\dot{g}(t)$ can be written

$$
f_{\dot{g}}(\dot{g} \mid d=z)=\mathcal{E}\left[f_{\dot{g}}\left(\dot{g} \mid d=z, \epsilon_{d}\right)\right]
$$

where $\mathcal{E}[\cdot]$ is the statistical expectation operator. The advantage of this formulation is that with $d(t)$ and $\epsilon_{d}(t)$ fixed, $\dot{g}(t)$ is a Gaussian. In Appendix I we show that substituting (7) and the Gaussian PDFs for $d(t)$ and $g(t)$ into (5) and (4) gives the LCR result

$$
\begin{aligned}
\operatorname{LCR}_{g}(T)=\frac{\sqrt{a b}}{\pi^{2} \sigma_{s}} \int_{0}^{\infty} \exp [ & -\frac{(T+\gamma \log z)^{2}}{2 \sigma_{s}^{2}} \\
& \left.-\frac{\left(z-d_{0}\right)^{2}}{2 \sigma_{m}^{2}}-\frac{\gamma^{2}}{4 b \sigma_{s}^{2} z^{2}}\right] d z
\end{aligned}
$$

\section{B. $M M 2$}

The approach for MM2 follows the same general scheme as for MM1 but is considerably more complex due to the twodimensional nature of the mobility model. In Appendix II we show that

$$
\dot{d}(t)=\sqrt{a} \frac{x(t) \epsilon_{x}(t)+y(t) \epsilon_{y}(t)}{\sqrt{x^{2}(t)+y^{2}(t)}}
$$

and

$$
\dot{X}(t)=\sqrt{2 a} \epsilon_{x}(t) \sqrt{\epsilon_{x}^{2}(t)+\epsilon_{y}^{2}(t)}
$$

Since $g(t)=X(t)-\gamma \log d(t)$ and $\dot{g}(t)=\dot{X}(t)-\gamma \dot{d}(t) / d(t)$ we see that conditioning on $x(t), y(t), \epsilon_{x}(t), \epsilon_{y}(t)$ leaves $g(t)$ and $\dot{g}(t)$ as independent variables. Hence, we can write

$$
\begin{array}{r}
f_{g, \dot{g}}(T, \dot{g})=\int_{-\infty}^{\infty} \ldots \int_{-\infty}^{\infty} f_{g}(T \mid x, y) f_{\dot{g}}\left(\dot{g} \mid x, y, \epsilon_{x}, \epsilon_{y}\right) \\
f\left(x, y, \epsilon_{x}, \epsilon_{y}\right) d x d y d \epsilon_{x} \epsilon_{y}
\end{array}
$$

where $f(\cdot, \cdot, \cdot, \cdot)$ is the joint PDF of $x(t), y(t), \epsilon_{x}(t), \epsilon_{y}(t)$. Note that both $f_{g}(T \mid x, y)$ and $f_{\dot{g}}\left(\dot{g} \mid x, y, \epsilon_{x}, \epsilon_{y}\right)$ are Gaussian PDFs. Also, since $x(t), y(t), \epsilon_{x}(t)$ and $\epsilon_{y}(t)$ are independent Gaussians, $f(\cdot, \cdot, \cdot, \cdot)$ can be broken down into four separate Gaussian PDFs. Substituting (11) into (4) leads after considerable algebraic manipulation to the desired result. Very brief details can be found in Appendix 2 and the final result is given by

$$
\begin{array}{r}
\operatorname{LCR}_{g}(T)=\frac{1}{\sqrt{2 \pi} \sigma_{s}} \int_{0}^{\infty} \exp \left[-\frac{(T+\gamma \log z)^{2}}{2 \sigma_{s}^{2}}\right] \\
\left(\mathcal{B}_{1}(z)+\mathcal{B}_{2}(z)\right) f_{d}(z) d z
\end{array}
$$

where

$$
\begin{gathered}
\mathcal{B}_{1}(z)=\sigma_{s} \sigma_{m} \sqrt{a b} \exp \left[-\frac{\gamma^{2}}{8 b \sigma_{s}^{2} z^{2}}\right] I_{0}\left(\frac{\gamma^{2}}{8 b \sigma_{s}^{2} z^{2}}\right), \\
\mathcal{B}_{2}(z)=\frac{\gamma \sigma_{m} \sqrt{a}}{2 z \sqrt{\pi}}\left\{\Phi\left(\frac{\gamma}{2 \sqrt{b} \sigma_{s} z}\right)-\Phi\left(-\frac{\gamma}{2 \sqrt{b} \sigma_{s} z}\right)\right\}, \\
f_{d}(z)=\frac{z}{\sigma_{m}^{2}} \exp \left[\frac{-\left(\lambda+z^{2} / \sigma_{m}^{2}\right)}{2}\right] I_{0}\left(z \sqrt{\lambda} / \sigma_{m}\right),
\end{gathered}
$$

and $\lambda=\left(x_{0}^{2}+y_{0}^{2}\right) / \sigma_{m}^{2}$. The functions $I_{0}(\cdot)$ and $\Phi(\cdot)$ are the zeroth order modified Bessel function and the standard Gaussian cumulative distribution function respectively.

\section{Simulation Results And Discussion}

In Figs. 2 and 3 we plot the LCR curve for MM1 under two scenarios. In both cases an average distance of $75 \mathrm{~m}$ between $\mathrm{CR}$ and PU is assumed with an average power of $5 \mathrm{~dB}$. The path loss exponent is 3.5 and $a=1$. In Fig. 2, $\sigma_{m}=5, \sigma_{\mathrm{dB}}=4.3 \mathrm{~dB}$ and $b=0.0142$ whereas in Fig. $3 \sigma_{m}=20, \sigma_{\mathrm{dB}}=7.5 \mathrm{~dB}$ and $b$ is in $(0.005,0.0142,0.1)$. These settings correspond to an urban scenario [14]. The LCR result is affected by the parameter $a$ only through scaling. Hence the choice of $a=1$ gives a normalized curve. The increased values of $\sigma_{m}$ and $\sigma_{\mathrm{dB}}$ result in a much broader LCR curve in Fig. 3. As can be seen, for both curves the analytical results are verified by simulation. Note that the effect of parameter $b$ is also a simple scaling. From (8) we see that $\sqrt{b}$ multiplies the LCR but $b$ also appears in the exponent term. The exponential term in $b$, however, has little effect since $z^{-2}$ is small around $d_{0}$ where most of the probability lies. Hence, the effect of $b$ is primarily due to the scaling term, $\sqrt{b}$. Also plotted in Figs. 2 and 3 are the best fitting Gaussian curves to approximate the LCR. The excellent agreement is due to the fact that $g(t)=X(t)-\log (d(t)), X(t)$ is exactly Gaussian and $\log (d(t))$ is unimodal and approximately symmetric. As a 


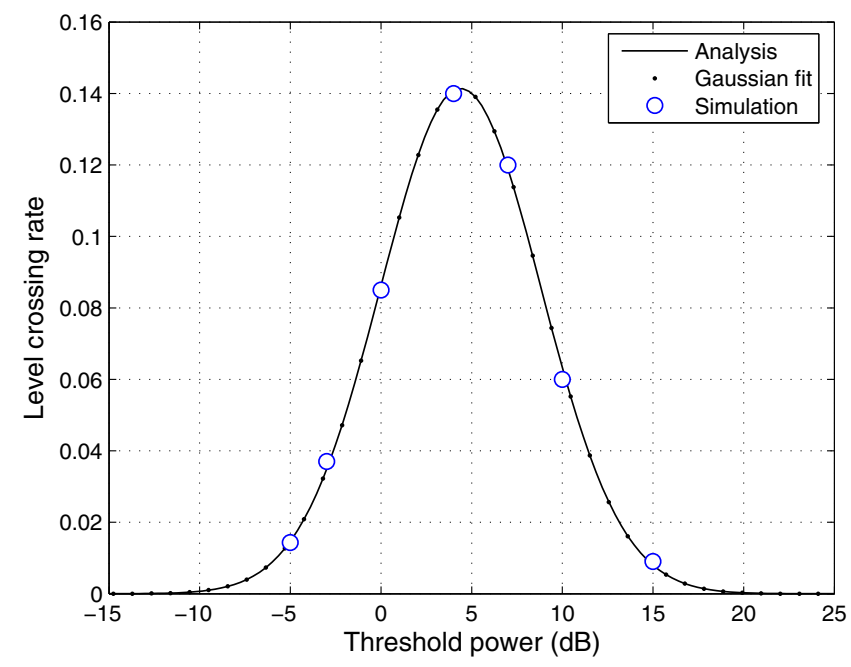

Fig. 2. Level crossing rate versus threshold power for MM1.

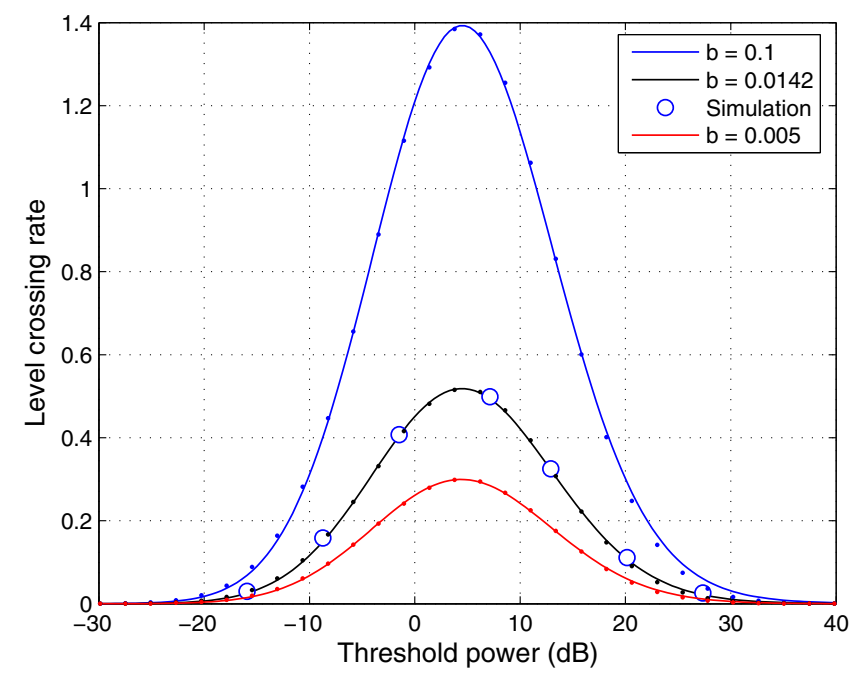

Fig. 3. Level crossing rate versus threshold power for MM1. The Gaussian fitted curve is shown by "dots".

result, $g(t)$, the sum of these variables, is also approximately Gaussian. Finally, we note that the LCR of a Gaussian process has a Gaussian shape, hence the proximity of the two curves. Similar results will occur with lognormal shadowing and any mobility model as long as the log-distance distribution is not strongly non-Gaussian. This makes the analysis much more widely applicable, which is useful as the mobility models presented here are rather simple and have limited scope. A general approach could use a Gaussian approximation to $g(t)$, for which LCR results are already known. The problem then simplifies to the computation of the mean, variance and ACF of the approximate Gaussian process. It is to be expected that this approach will remain tractable for a far wider class of mobility models.

Next, we consider the MM2 model. The parameters used are the same as for Fig. 3 with $b=0.0142$ and $\sqrt{x_{0}^{2}+y_{0}^{2}}=75$. As shown in Fig. 4 the analytical result is verified by simu-

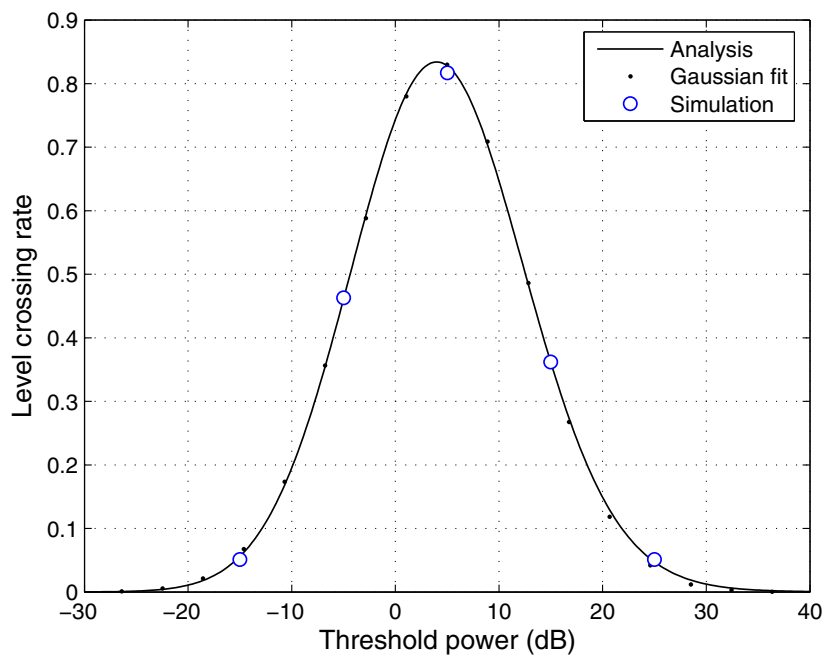

Fig. 4. Level crossing rate versus threshold power for MM2.

lation and a Gaussian approximation is excellent once more. It is worth noting that the analytical results are very rapid and numerically robust whereas the simulations require large numbers of iterations before accurate estimates are returned.

Note that for MM1 the distance is Gaussian, whereas for MM2 distance is the square root of a non-central chi-squared variable. Hence, as discussed above, despite variation in the mobility models and the corresponding distance distributions the basic Gaussian shape of the LCR curve remains.

\section{Conclusions}

We have derived exact LCR results for the classic lognormal shadowing model which caters for temporal variation both in shadowing and in distance. Our analysis demonstrates that a simple Gaussian approximation may be appropriate as a general methodology. Hence, for complex mobility patterns the problem reduces to finding second order statistics which suffice to compute a Gaussian LCR. The effect of increasing variance in distance and shadow fading is to spread the LCR curves and changes in the ACFs of the mobility and shadowing models scale the LCR. The analysis has direct application to CR systems where LCRs across detection thresholds at the CR and allowable interference thresholds at the PU are important for performance analysis.

\section{APPENDiX A}

\section{LCR CALCULATION FOR MM1}

From (7) we can write $f_{\dot{g}}(\dot{g} \mid d=z)$ as

$$
\frac{1}{4 \pi \epsilon_{d} \sigma_{m} \sigma_{s} \sqrt{a b}} \int_{-\infty}^{\infty} \exp \left[-\frac{\left(\dot{g}+\gamma \epsilon_{d} / z\right)^{2}}{4 \epsilon_{d}^{2} b \sigma_{s}^{2}}-\frac{\epsilon_{d}^{2}}{4 a \sigma_{m}^{2}}\right] d \epsilon_{d}
$$


Also, both $f_{g}(T \mid d=z)$ and $f_{d}(z)$ in (5) are Gaussian, so the LCR can be written as

$$
\begin{aligned}
\operatorname{LCR}_{g}(T) & =\frac{1}{8 \pi^{2} \sigma_{s}^{2} \sigma_{m}^{2}\left|\epsilon_{d}\right| \sqrt{a b}} \int_{0}^{\infty} \int_{0}^{\infty} \int_{-\infty}^{\infty} \dot{g} \\
& \times \exp \left[-\frac{\left(\dot{g}+\gamma \epsilon_{d} / z\right)^{2}}{4 \epsilon_{d}^{2} b \sigma_{s}^{2}}-\frac{\epsilon_{d}^{2}}{4 b \sigma_{m}^{2}}\right. \\
& \left.-\frac{(T+\gamma \log z)^{2}}{2 \sigma_{s}^{2}}-\frac{\left(z-d_{0}\right)^{2}}{2 \sigma_{m}^{2}}\right] d \epsilon_{d} d z d \dot{g}
\end{aligned}
$$

Next, we reorder the integrals so that the integration over $\dot{g}$ is performed first. We use the result,

$$
\begin{aligned}
\frac{1}{2 \epsilon_{d} \sigma_{s} \sqrt{\pi b}} & \int_{0}^{\infty} \dot{g} \exp \left[-\frac{\left(\dot{g}+\gamma \epsilon_{d} / z\right)^{2}}{4 \epsilon_{d}^{2} b \sigma_{s}^{2}}\right] d \dot{g}=\frac{\left|\epsilon_{d}\right| \sqrt{2 b} \sigma_{s}}{\sqrt{2 \pi}} \\
& \times \exp \left[-\frac{\gamma^{2}}{4 b \sigma_{s}^{2} z^{2}}\right]-\frac{\gamma \epsilon_{d}}{z}\left[1-\Phi\left(\frac{\gamma}{2 b \sigma_{s} z}\right)\right]
\end{aligned}
$$

The next integration over $\epsilon_{d}$ simplifies since

$$
\int_{-\infty}^{\infty} \epsilon_{d} \exp \left(-\frac{\epsilon_{d}^{2}}{4 a \sigma_{m}^{2}}\right) d \epsilon_{d}=0
$$

and

$$
\int_{-\infty}^{\infty}\left|\epsilon_{d}\right| \exp \left(-\frac{\epsilon_{d}^{2}}{4 a \sigma_{m}^{2}}\right) d \epsilon_{d}=4 a \sigma_{m}^{2}
$$

Substituting these results in (14) gives the desired LCR in (8).

\section{APPENDIX B}

\section{LCR CALCULATION FOR MM2}

For MM2 we require a slightly different version of (6). Let $w(t)$ be the distance moved by the terminal at time $t$. The shadow fading varies with $w(t)$ rather than $d(t)$. Hence we define

$$
\dot{X}(t)=\frac{\partial w(t)}{\partial t} \frac{\partial X(t)}{\partial w(t)}=\dot{w}(t) \frac{\partial X(t)}{\partial w(t)}
$$

As before we define $\frac{\partial X(t)}{\partial w(t)}=\epsilon_{X}(t)$ where $\epsilon_{X}(t)$ is a white Gaussian noise process with zero mean and variance $2 b \sigma_{s}^{2}$. Next we require $\dot{d}(t)$ (to compute $\dot{g}(t)$ ) and $\dot{w}(t)$ (to compute $\dot{X}(t))$. The distance travelled in $[t, t+\tau]$ is $w(t+\tau)-w(t)$ which is given by

$$
\begin{aligned}
& \left(\left[x_{0}+\rho_{m}(\tau)\left(x(t)-x_{0}\right)+\sqrt{1-\rho_{m}^{2}(\tau)}\right.\right. \\
& \left.\epsilon_{x}(t+\tau)-x(t)\right]^{2}+\left[y_{0}+\rho_{m}(\tau)\left(y(t)-y_{0}\right)\right. \\
& \left.\left.+\sqrt{1-\rho_{m}^{2}(\tau)} \epsilon_{y}(t+\tau)-y(t)\right]^{2}\right)^{\frac{1}{2}}
\end{aligned}
$$

Taking the limit of $(w(t+\tau)-w(t)) / \tau$ as $\tau \rightarrow 0$ gives

$$
\dot{w}(t)=\sqrt{4 a} \sqrt{\epsilon_{x}^{2}(t)+\epsilon_{y}^{2}(t)}
$$

Similarly, over the interval $[t, t+\tau]$ the change in $d(t), d(t+$ $\tau)-d(t)$, is given by

$$
\begin{aligned}
& \left(\left[x_{0}+\rho_{m}(\tau)\left(x(t)-x_{0}\right)+\sqrt{1-\rho_{m}^{2}(\tau)} \epsilon_{x}(t+\tau)\right]^{2}\right. \\
& \left.+\left[y_{0}+\rho_{m}(\tau)\left(y(t)-y_{0}\right)+\sqrt{1-\rho_{m}^{2}(\tau)} \epsilon_{y}(t+\tau)\right]^{2}\right)^{\frac{1}{2}} \\
& -\sqrt{x^{2}(t)+y^{2}(t)}
\end{aligned}
$$

Taking the limit of $(d(t+\tau)-d(t)) / \tau$ as $\tau \rightarrow 0$ gives

$$
\dot{d}(t)=\sqrt{2 a} \frac{x(t) \epsilon_{x}(t)+y(t) \epsilon_{y}(t)}{\sqrt{x^{2}(t)+y^{2}(t)}}
$$

Hence, from (18), (20) and (22) we have

$$
\begin{aligned}
\dot{g}(t) & =\dot{X}(t)-\gamma \frac{\dot{d}(t)}{d t} \\
& =\sqrt{4 a} \epsilon_{X}(t) \sqrt{\epsilon_{x}^{2}(t)+\epsilon_{y}^{2}(t)} \\
& -\gamma \sqrt{2 a} \frac{x(t) \epsilon_{x}(t)+y(t) \epsilon_{y}(t)}{x^{2}(t)+y^{2}(t)}
\end{aligned}
$$

With $\dot{d}(t)$ and $\dot{g}(t)$ given by (22) and (23) we can rewrite (11) so that (4) gives

$$
\begin{aligned}
\operatorname{LCR}_{g}(T) & =\frac{1}{4 \pi \sigma_{s}^{2} \sqrt{a b}} \int_{0}^{\infty} \dot{g} \int_{0}^{\infty} \cdots \int_{0}^{\infty} \frac{1}{\sqrt{\epsilon_{x}^{2}+\epsilon_{y}^{2}}}(24) \\
& \times \exp \left[-\frac{(T+\gamma \log d)^{2}}{2 \sigma_{s}^{2}}\right] \exp \left[-\frac{(\dot{g}+\gamma \dot{d} / d)^{2}}{8 a b \sigma_{s}^{2}\left(\epsilon_{x}^{2}+\epsilon_{y}^{2}\right)}\right] \\
& \times f\left(x, y, \epsilon_{x}, \epsilon_{y}\right) d x d y d \epsilon_{x} d \epsilon_{y} d \dot{g} .
\end{aligned}
$$

Rearranging the order of integration in (24), the integral over $\dot{g}$ can be done first in closed-form. The following integrals over $\epsilon_{x}, \epsilon_{y}$ can be performed by using the polar coordinates, $\epsilon_{x}=r \cos \theta, \epsilon_{y}=r \sin \theta$. The final resulting integral is solely a function of $d=\sqrt{x^{2}+y^{2}}$ and gives the final result in (12).

\section{ACKNOWLEDGEMENT}

This research is supported under the Australian Research Council's Discovery funding scheme (DP0774689).

\section{REFERENCES}

[1] Federal Communications Commission (FCC), "Facilitating opportunities for flexible, efficient, and reliable spectrum use employing cognitive radio technologies," ET Docket No. 03-108, Mar. 2005.

[2] Cognitive Radio Technology, [Online] Available: http://www.ofcom.org uk/research/technology/overview/emer_tech/cograd/cograd_main.pdf

[3] J. Mitola III, Cognitive radio: An integrated agent architecture for software defined radio, Ph.D Thesis, KTH Royal Institute of Technology, Sweden, May 2000.

[4] S. Haykin, "Cognitive radio: Brain-empowered wireless communications," IEEE J. Select. Areas Commun., vol. 23, pp. 201-220, Feb. 2005.

[5] F. K. Jondral, "Cognitive radio: A communications engineering view," IEEE Wireless Commun. Mag., vol. 14, pp. 28-33, Aug. 2007.

[6] I. F. Akyildiz, W.-Y. Lee, M. C. Vuran and S. Mohanty, "Next generation/dynamic spectrum access/cognitive radio wireless networks: A survey," Computer Networks, vol. 50, pp. 2127-2159, 2006. 
[7] P. J. Kolodzy, "Interference temperature: A metric for dynamic spectrum utilization," International Journal on Network Management, vol. 16, pp. 103-113, 2006.

[8] F. Digham, M. Alouini and M. Simon, "On the energy detection of unknown signals over fading channels," in Proc. IEEE ICC 2003, Anchorage, AK, May 2003, pp. 3575-3579.

[9] A. Pandharipande and J.-P. M. G. Linnartz, "Performance analysis of primary user detection in a multiple antenna cognitive radio, in Proc. IEEE ICC 2007, Glasgow, Scotland, pp. 6482-6486.

[10] S. O. Rice, "Statistical properties of a sine wave plus noise," Bell System Tech. J., vol. 27, pp. 109157, Jan. 1948

[11] W. C. Jakes, Microwave Mobile Communications. New York: Wiley, 1974.

[12] P. Ivanis, D. Drajic and B. Vucetic, "Level crossing rates of MIMOMRC Ricean channels and their implications on adaptive systems," in
Proc. IEEE ICC 2007, Glasgow, Scotland, June 2007, pp. 744-749.

[13] P. J. Smith, P.-H. Kuo and L. M. Garth, "Level crossing rates for MIMO channel eigenvalues: Implications for adaptive systems," in Proc. IEEE ICC 2005, Seoul, South Korea, May 2005, pp. 2242-2446.

[14] M. Pätzold and K. Yang, "An exact solution for the level-crossing rate of shadow fading processes modelled by using the sum-of-sinusoids principle," in Proc. IEEE WPMC 2006, San Diego, CA, Sept. 2006, pp. 188-193.

[15] H. Cramer and M. R. Leadbetter, Stationary and Related Stochastic Processes. New York: Wiley, 1967.

[16] M. Gudmundson, "Correlation model for shadow fading in mobile radio systems," Electron. Lett., vol. 27, pp. 21452146, Nov. 1991.

[17] N. C. Beaulieu, A. A. Abu-Dayya and P. J. McLane, "Estimating the distribution of a sum of independent lognormal random variables," IEEE Trans. Commun., vol. 43, pp. 2869-2873, Dec. 1995. 